\title{
Monotone iterative technique for non-autonomous semilinear differential equations with nonlocal condition
}

https://doi.org/10.1515/dema-2019-0005

Received August 2, 2018; accepted December 17, 2018

\begin{abstract}
The objective of this article is to discuss the existence and uniqueness of mild solutions for a class of non-autonomous semilinear differential equations with nonlocal condition via monotone iterative method with upper and lower solutions in an ordered complete norm space $\mathrm{X}$, using evolution system and measure of noncompactness.
\end{abstract}

Keywords: evolution system, monotone iterative technique, lower and upper solutions, Kuratowski measure of noncompactness

MSC: $34 \mathrm{G} 20,34 \mathrm{~K} 30$

\section{Introduction}

In this work, we will apply monotone iterative method to find the existence and uniqueness of mild solutions for the following non-autonomous system in an ordered Banach space X:

$$
\begin{aligned}
x^{\prime}(t)+\mathbb{A}(t) x(t) & =f(t, x(t)), \quad t \in(0, b], \\
x(0) & =\sum_{i=1}^{k} \xi_{i} x\left(t_{i}\right)+x_{0},
\end{aligned}
$$

where $\mathbb{A}(t): D(\mathbb{A}(t)) \subset X \rightarrow X$ is a linear operator, $J=[0, b], f$ is a given function from $J \times X$ to $X$ satisfying certain assumptions, $t_{i} \in(0, b)$, for $i<j, t_{i}<t_{j}, 0 \neq \xi_{i} \in \mathbb{R}, i=1,2, \ldots, k \in \mathbb{N}, x_{0} \in X$.

Nonlocal condition is a generalization of classical initial condition which is more effective to produce better results in the application of physical problems rather than classical initial condition. The existence results for nonlocal Cauchy problem was first studied by Byszewski [1]. To describe the diffusion phenomenon of a small amount of gas in a transparent tube, Deng [2] used the nonlocal condition. In [3], authors discussed the existence of mild solutions for a class of fractional evolution equations with nonlocal integral conditions with the help of compact semigroup, approximation technique and Schauder fixed point theorem.

Monotone iterative technique is an effective method to find the existence and uniqueness of mild solutions. Using this method, we get monotone sequences of approximate solutions that converge to maximal and minimal mild solutions. Du [4], first used this technique to find extremal mild solutions for a differential equation. The technique has been used for autonomous system till now. Chen and Li [5] used monotone iterative technique to establish the existence and uniqueness of mild solutions for a semilinear differential equation with nonlocal condition. In [6], Chen and $\mathrm{Mu}$ discussed the existence and uniqueness of mild solutions for a semilinear impulsive integro-differential equation by using monotone iterative method. $\mathrm{Mu}$ [7] studied the existence and uniqueness of mild solutions for a class of fractional evolution equations with the

^Corresponding Author: Arshi Meraj: Indian Institute of Technology Roorkee, India; E-mail: arshimeraj@gmail.com Dwijendra Narain Pandey: Indian Institute of Technology Roorkee, India; E-mail: dwij.iitk@gmail.com 
help of monotone iterative method. In [8], Mu and Li investigated the existence and uniqueness of mild solutions for an impulsive fractional differential equation by using monotone iterative technique. Chen et al. [9] studied the existence of mild solutions for a nonlocal impulsive semilinear evolution equation by using perturbation method and monotone iterative technique. In [10], authors considered a fractional differential equation with mixed monotone nonlocal conditions to investigate the existence of mild solutions with the help of coupled lower and upper mild $L$-quasi solutions and monotone iterative technique. Kamaljeet [11] and Renu [12] used monotone iterative method to discuss the existence and uniqueness of extremal mild solutions for nonlocal fractional differential equations with finite delay and for neutral fractional differential equations with infinite delay, respectively. Chen et al. [13] used perturbation method and iterative technique to establish the existence of mild solutions for a class of evolution equations with non-instantaneous impulses.

The existence of mild solutions of a non-autonomous integro-differential equations with nonlocal conditions by using the theory of evolution families, Banach contraction principle and Schauder's fixed point theorem is studied by Yan [14]. Haloi et al. [15] studied existence, uniqueness and asymptotic stability of non-autonomous differential equations with deviated arguments via Banach fixed point theorem and theory of analytic semigroup. In [16], Alka et al. established the existence and uniquenss of mild solutions for non-autonomous instantaneous impulsive differential equations with iterated deviating arguments by using analytic semigroup theory and Banach fixed point theorem. Borai [17] first derived the sufficient conditions for the existence of solution for a fractional non-autonomous Cauchy problem. Fang Li [18] discussed the existence of mild solutions for fractional non-autonomous integro-differential equations with nonlocal conditions. Chen et al. [19] studied the existence of mild solutions for a class of fractional non-autonomous evolution equations with delay by using analytic semigroup, measure of noncompactness and fixed point theorem with respect to $k$-set contractive operator. Nieto [20] established the sufficient conditions for the existence of mild solutions for a second order non-autonomous evolution equation having nonlocal conditions by using measure of noncompactness and Tikhonoff fixed point theorem. In [21], Chen et al. established the existence of mild solutions for a non-autonomous fractional differential equation by using the theory of evolution families and fixed point theorem with respect to $k$ - set contractive operator. In [22], authors first studied the local existence of mild solutions, then obtained a blowup alternative result for fractional non-autonomous integrodifferential equation of Volterra type.

To the best of our knowledge, there is no work yet reported on the existence and uniqueness of mild solutions for non-autonomous differential equations by using monotone iterative method. Motivated by this fact, in this article, we consider a non-autonomous differential system with nonlocal condition to investigate the sufficient conditions for the existence and uniqueness of mild solutions with the help of monotone iterative technique.

We organize the article as following: In section 2, we will recall some basic definitions, notations and theorems. In section 3, we will study the existence of extremal mild solutions for the system (1.1)-(1.2), and also we will show the uniqueness of extremal mild solutions. Finally, in section 4, we will present an example to illustrate our results.

\section{Preliminaries}

Now, we recall some basic theory which is required for our main results.

Let $(X,\|\cdot\|, \leqslant)$ be a partially ordered complete norm space, $\mathcal{P}=\{x \in X \mid \quad x \geqslant 0\}$ ( 0 is the zero of $X)$ is a positive cone of $X$. The cone $\mathcal{P}$ is called normal if there is a real number $\mathcal{N}>0$ such that $0 \leqslant x_{1} \leqslant x_{2} \Rightarrow$ $\left\|x_{1}\right\| \leqslant \mathcal{N}\left\|x_{2}\right\|$, for all $x_{1}, x_{2} \in X$, the smallest value of such $\mathcal{N}$ is called normal constant. Let $\mathbf{C}(J, X)$ be space of all continuous maps from $J$ to $X$, with sup norm. For $x_{1}, x_{2} \in \mathbf{C}(J, X), x_{1} \leqslant x_{2} \Leftrightarrow x_{1}(t) \leqslant x_{2}(t), t \in J$, the interval $[v, \omega]=\{x \in \mathbf{C}(J, X): v \leqslant x \leqslant \omega\}$ in $\mathbf{C}(J, X)$, and $[v(t), \omega(t)]=\{x \in X: v(t) \leqslant x \leqslant \omega(t)\}(t \in J)$ in $X$. Let us denote $C^{1}(J, X)=\left\{x \in \mathbf{C}(J, X): x^{\prime}\right.$ exists on $\left.J, x^{\prime} \in \mathbf{C}(J, X), x(t) \in D(\mathbb{A})(t \geqslant 0)\right\}$. 
Definition 2.1. [23] Let $X$ be a Banach space. A two parameter family of bounded linear operators $\mathbb{U}(t, s), 0 \leqslant$ $s \leqslant t \leqslant b$ on $X$ is called an evolution system, if the following conditions are satisfied:

1. $\mathbb{U}(s, s)=I$, where $I$ is the identity operator.

2. $\mathbb{U}(t, r) \mathbb{U}(r, s)=\mathbb{U}(t, s)$ for $0 \leqslant s \leqslant r \leqslant t \leqslant b$.

3. $(t, s) \rightarrow \mathbb{U}(t, s)$ is strongly continuous for $0 \leqslant s \leqslant t \leqslant b$.

For the family of linear operators $\{\mathbb{A}(t): t \in J\}$ on $X$, we impose the following assumptions:

(A1) The domain of $\mathbb{A}(t)$ is dense and independent of $t$, and $\mathbb{A}(t)$ is a closed operator.

(A2) The resolvent of $\mathbb{A}(t)$ exists for $\operatorname{Re}(\vartheta) \leqslant 0, t \in J$, and there exists a positive constant $\varsigma$ such that $\|\mathcal{R}(\vartheta ; t)\| \leqslant \frac{\varsigma}{|\vartheta|+1}$.

(A3) There are positive constants $K$, and $\rho \in(0,1]$ such that $\left\|\left[\mathbb{A}\left(\tau_{1}\right)-\mathbb{A}\left(\tau_{2}\right)\right] \mathbb{A}^{-1}\left(\tau_{3}\right)\right\| \leqslant K\left|\tau_{1}-\tau_{2}\right|^{\rho}$ for any $\tau_{1}, \tau_{2}, \tau_{3} \in J$.

The above assumptions imply that, $-\mathbb{A}(t)$ generates a unique evolution system $\{\mathbb{U}(t, s): 0 \leqslant s \leqslant t \leqslant b\}$ of bounded linear operators on $X$, which satisfies the following properties (see [23, 24]):

(i) $\mathbb{U}\left(t_{1}, t_{2}\right) \in B(X)$ the space of bounded linear operators on $X$, and is continuous strongly for $0 \leqslant t_{2} \leqslant$ $t_{1} \leqslant b$.

(ii) $\mathbb{U}\left(t_{1}, t_{2}\right) x \in D(\mathbb{A}), x \in X, 0 \leqslant t_{2} \leqslant t_{1} \leqslant b$.

(iii) $\mathbb{U}\left(t_{1}, t_{2}\right) U\left(t_{2}, t_{3}\right)=\mathbb{U}\left(t_{1}, t_{3}\right), 0 \leqslant t_{3} \leqslant t_{2} \leqslant t_{1} \leqslant b$.

(iv) $\mathbb{U}(\tau, \tau)=I$ the identity operator on $X$, for each $\tau \in J$.

(v) There is a positive constant $\mathcal{M}$ such that $\left\|\mathbb{U}\left(t_{1}, t_{2}\right)\right\| \leqslant \mathcal{M}, 0 \leqslant t_{2} \leqslant t_{1} \leqslant b$.

(vi) For each fixed $s,\{\mathbb{U}(\tau, s), s \leqslant \tau\}$ is continuous in $\tau$ in uniform operator topology.

(vii) The derivative $\frac{\partial \mathbb{U}(\tau, s)}{\partial \tau}$ exists in strong operator topology for $0 \leqslant s<\tau \leqslant b$, and strongly continuous in $\tau$, where $0 \leqslant s<\tau \leqslant b$.

(viii) $\frac{\partial \mathbb{U}(\tau, s)}{\partial \tau}+\mathbb{A}(\tau) \mathbb{U}(\tau, s)=0,0 \leqslant s<\tau \leqslant b$.

Lemma 2.2. [24, Lemma 14.1] Let assumptions (A1)-(A3) hold. If $0 \leqslant \gamma \leqslant 1,0 \leqslant \beta \leqslant \delta<1+\alpha, 0<\delta-\gamma \leqslant 1$, then for any $0 \leqslant \tau<t<t+\triangle t \leqslant t_{0}, 0 \leqslant \zeta \leqslant t_{0}$,

$$
\left\|\mathbb{A}^{\gamma}(\zeta)[\mathbb{U}(t+\triangle t, \tau)-\mathbb{U}(t, \tau)] \mathbb{A}^{-\beta}(\tau)\right\| \leqslant C(\beta, \gamma, \delta)(\triangle t)^{\delta-\gamma}|t-\tau|^{\beta-\delta} .
$$

Definition 2.3. An evolution system $\mathbb{U}(t, s)$ is called positive if $\mathbb{U}(t, s) y \geqslant 0$, for $y \in \mathcal{P}$ and $0 \leqslant s \leqslant t \leqslant b$.

Definition 2.4. $\omega_{0} \in C^{1}(J, X)$ is called lower solution for the system (1.1)-(1.2), if

$$
\begin{aligned}
\omega_{0}^{\prime}(t)+\mathbb{A}(t) \omega_{0}(t) & \leqslant f\left(t, \omega_{0}(t)\right), \quad t \in(0, b], \\
\omega_{0}(0) & \leqslant \sum_{i=1}^{k} \xi_{i} \omega_{0}\left(t_{i}\right)+x_{0} .
\end{aligned}
$$

If the inequalities of (2.1) are opposite, solution is known as upper solution.

Theorem 2.5. [23, 24] Suppose that the assumptions ( $A 1)$-(A3) hold and $f$ satisfies uniform Hölder continuity on $J$ with exponent $\beta \in(0,1]$, then unique solution of the following linear Cauchy problem

$$
\begin{aligned}
x^{\prime}(t)+\mathbb{A}(t) x(t) & =f(t), \quad t \in(0, b], \\
x(0) & =x_{0} \in X,
\end{aligned}
$$

is

$$
x(t)=\mathbb{U}(t, 0) x_{0}+\int_{0}^{t} \mathbb{U}(t, s) f(s) d s
$$


Now, we state the definition and some properties of Kuratowski measure of noncompactness. For more details, we refer [25] and [26].

Definition 2.6. If $\mathbb{Y}$ is a Banach space and $M(\mathbb{Y})$ is a collection of subsets of $\mathbb{Y}$, which are bounded, then the function $\mu: M(\mathbb{Y}) \rightarrow[0, \infty)$ as following

$$
\mu(S)=\inf \left\{\varepsilon>0: S \subset \cup_{j=1}^{n} S_{j}, \operatorname{diam}\left(S_{j}\right)<\varepsilon(j=1,2, \ldots, n \in \mathbb{N})\right\}
$$

is known as Kuratowski measure of noncompactness.

Lemma 2.7. If $\mathbb{X}_{1}$ and $\mathbb{X}_{2}$ be Banach spaces and $C, D \subset \mathbb{X}_{1}$ be bounded, then the following properties are satisfied:

(i) $D$ is precompact if and only if $\mu(D)=0$.

(ii) $\mu(C+D) \leqslant \mu(C)+\mu(D)$.

(iii) $\varphi: \operatorname{dom}(\varphi) \subset \mathbb{X}_{1} \rightarrow \mathbb{X}_{2}$ satisfies Lipschitz continuity with constant $L$, then $\mu(\varphi(S)) \leqslant L \mu(S), S \subset$ $\operatorname{dom}(\varphi)$ is bounded.

Lemma 2.8. If $\mathbb{Y}$ is a Banach space, and $S \subset \mathbf{C}(J, \mathbb{Y}), S(t)=\{x(t): x \in S\}(t \in J)$. Then boundedness and equicontinuity of $S$ in $\mathbf{C}(J, \mathbb{Y})$ implies that $\mu(S(t))$ is continuous on $J$, moreover $\mu(S)=\max _{t \in J} \mu(S(t))$.

Lemma 2.9. Suppose $\mathbb{X}$ is complete norm space and $\left\{u_{n}\right\} \subset \mathbf{C}(J, \mathbb{X})$ is a bounded sequence. Then, $\mu\left(\left\{u_{n}(t)\right\} \in\right.$ $L^{1}(J, \mathbb{X})$, moreover

$$
\mu\left(\left\{\int_{0}^{t} u_{n}(s) d s\right\}_{n=1}^{\infty}\right) \leqslant 2 \int_{0}^{t} \mu\left(\left\{u_{n}(s)\right\}_{n=1}^{\infty}\right) d s .
$$

\section{Main results}

First, we will derive the expression of mild solution for the problem (1.1)-(1.2), then the existence as well as uniqueness of extremal mild solutions will be discussed. Let us introduce our basic assumptions:

(Ho) The evolution system $\mathbb{U}(t, s)$ is positive.

(H1) $\xi_{i}>0, i=1,2, \ldots, k$ and $\mathcal{M} \sum_{i=1}^{k} \xi_{i}<1$.

Assumption (H1) implies that

$$
\left\|\sum_{i=1}^{k} \xi_{i} \mathbb{U}\left(t_{i}, 0\right)\right\| \leqslant \mathcal{M} \sum_{i=1}^{k} \xi_{i}<1,
$$

by operator spectrum theorem, we know that

$$
\mathcal{B}:=\left(I-\sum_{i=1}^{k} \xi_{i} \mathbb{U}\left(t_{i}, 0\right)\right)^{-1}
$$

exists, bounded and can be expressed as

$$
\mathcal{B}=\sum_{j=0}^{\infty}\left(\sum_{i=1}^{k} \xi_{i} \mathbb{U}\left(t_{i}, 0\right)\right)^{j} .
$$


Since $\mathbb{U}(t, s)$ is positive, so is $\mathcal{B}$ and

$$
\begin{aligned}
\|\mathcal{B}\| & \leqslant \sum_{j=0}^{\infty}\left\|\sum_{i=1}^{k} \xi_{i} \mathbb{U}\left(t_{i}, 0\right)\right\|^{j} \leqslant \sum_{j=0}^{\infty}\left(\mathcal{M} \sum_{i=1}^{k} \xi_{i}\right)^{j} \\
& \leqslant \frac{1}{1-\mathcal{M} \sum_{i=1}^{k} \xi_{i}} .
\end{aligned}
$$

Definition 3.1. A function $x \in \mathbf{C}(J, X)$ is said to be a mild solution of the problem (1.1)-(1.2), if $x$ satisfies the following integral equation:

$$
x(t)=\mathbb{U}(t, 0) \mathcal{B} x_{0}+\sum_{i=1}^{k} \xi_{i} \mathbb{U}(t, 0) \mathcal{B} \int_{0}^{t_{i}} \mathbb{U}\left(t_{i}, s\right) f(s, x(s)) d s+\int_{0}^{t} \mathbb{U}(t, s) f(s, x(s)) d s .
$$

Lemma 3.2. If the assumptions ( $A 1)$-( $A 3$ ) and (H1) hold and $f \in \mathbf{C}(J \times X, X)$, then the unique mild solution of (1.1)-(1.2) is given as

$$
x(t)=\mathbb{U}(t, 0) \mathcal{B} x_{0}+\sum_{i=1}^{k} \xi_{i} \mathbb{U}(t, 0) \mathcal{B} \int_{0}^{t_{i}} \mathbb{U}\left(t_{i}, s\right) f(s, x(s)) d s+\int_{0}^{t} \mathbb{U}(t, s) f(s, x(s)) d s .
$$

Proof. By Theorem 2.5, the mild solution of problem (1.1)-(1.2) can be expressed as

$$
x(t)=\mathbb{U}(t, 0) x(0)+\int_{0}^{t} \mathbb{U}(t, s) f(s, x(s)) d s .
$$

So,

$$
x\left(t_{i}\right)=\mathbb{U}\left(t_{i}, 0\right) x(0)+\int_{0}^{t_{i}} \mathbb{U}\left(t_{i}, s\right) f(s, x(s)) d s,
$$

for $i=1,2,3, \ldots, k$. By using (3.7) in (1.2), we get

$$
x(0)=\sum_{\substack{i=1 \\+x_{0}}}^{k} \xi_{i}\left[\mathbb{U}\left(t_{i}, 0\right) x(0)+\int_{0}^{t_{i}} \mathbb{U}\left(t_{i}, s\right) f(s, x(s))\right] d s
$$

Now, assumption (H1) and (3.1) imply that

$$
x(0)=\mathcal{B} x_{0}+\sum_{i=1}^{k} \xi_{i} \mathcal{B} \int_{0}^{t_{i}} \mathbb{U}\left(t_{i}, s\right) f(s, x(s)) d s .
$$

Hence (3.6) and (3.8) imply that (3.5) is satisfied.

Let us define $Q: \mathbf{C}(J, X) \rightarrow \mathbf{C}(J, X)$ as:

$$
Q x(t)=\mathbb{U}(t, 0) \mathcal{B} x_{0}+\sum_{i=1}^{k} \xi_{i} \mathbb{U}(t, 0) \mathcal{B} \int_{0}^{t_{i}} \mathbb{U}\left(t_{i}, s\right) f(s, x(s)) d s+\int_{0}^{t} \mathbb{U}(t, s) f(s, x(s)) d s .
$$

To prove that the system (1.1)-(1.2) has a mild solution, we need to show the operator $Q$ has a fixed point.

Theorem 3.3. Suppose $X$ is a partially ordered complete norm space with normal positive cone $\mathcal{P}$, the assumptions (H0), (H1) and (A1)-(A3) hold, $f$ is continuous from $J \times X \rightarrow X, x_{0} \in X$, and $\omega_{0}, v_{0} \in C^{1}(J, X)$ are lower and upper solutions respectively for (1.1)-(1.2). Moreover, assume the following: 
(H2) For $t \in J$, and $y_{1}, y_{2} \in X$ with $\omega_{0}(t) \leqslant y_{1} \leqslant y_{2} \leqslant v_{0}(t)$;

$$
f\left(t, y_{1}\right) \leqslant f\left(t, y_{2}\right) \text {. }
$$

(H3) There is a constant $\mathcal{L}>0$ such that for $t \in J$, and monotone sequence $\left\{x_{n}\right\}$ in $\left[\omega_{0}(t), v_{0}(t)\right]$;

$$
\mu\left(\left\{f\left(t, x_{n}\right)\right\}\right) \leqslant L \mu\left(\left\{x_{n}\right\}\right) .
$$

(H4) Suppose $\omega_{n}=Q \omega_{n-1}$ and $v_{n}=Q v_{n-1}, n \in \mathbb{N}$ with $\left\{\omega_{n}(0)\right\}$ and $\left\{v_{n}(0)\right\}$ are convergent.

Then, the system (1.1)-(1.2) has extremal mild solutions in the interval $\left[\omega_{0}, v_{0}\right]$, provided that $K:=2 M L b<1$.

Proof. Let us denote $D=\left[\omega_{0}, v_{0}\right]$. For any $x \in D,(H 2)$ implies

$$
f\left(t, \omega_{0}(t)\right) \leqslant f(t, x(t)) \leqslant f\left(t, v_{0}(t)\right) .
$$

Therefore, from the normality of $\mathcal{P}$ we get a constant $c>0$, such that

$$
\|f(t, x(t))\| \leqslant c, x \in D .
$$

First, we will prove that the map $\mathcal{Q}: D \rightarrow \mathbf{C}(J, X)$ is continuous. Let $\left\{x_{n}\right\}$ be a sequence in $D$ such that $x_{n} \rightarrow$ $x \in D$. Since $f$ is continuous, so $f\left(\varrho, x_{n}(\varrho)\right) \rightarrow f(\varrho, x(\varrho))$ for $\varrho \in J$, and from (3.10) we get that $\| f\left(\varrho, x_{n}(\varrho)\right)-$ $f(\varrho, x(\varrho)) \| \leqslant 2 c$. So, by Lebesgue dominated convergence theorem, we estimate

$$
\begin{gathered}
\left\|Q x_{n}(t)-Q x(t)\right\| \leqslant \sum_{i=1}^{k} \xi_{i}\|\mathbb{U}(t, 0)\|\|\mathcal{B}\| \int_{0}^{t_{i}}\left\|\mathbb{U}\left(t_{i}, \varrho\right)\right\|\left\|f\left(\varrho, x_{n}(\varrho)\right)-f(\varrho, x(\varrho))\right\| d \varrho \\
\quad+\int_{0}^{t}\|\mathbb{U}(t, \varrho)\|\left\|f\left(\varrho, x_{n}(\varrho)\right)-f(\varrho, x(\varrho))\right\| d \varrho . \\
\left\|Q x_{n}(t)-Q x(t)\right\| \leqslant \mathcal{M}^{2}\|\mathcal{B}\| \sum_{i=1}^{k} \xi_{i} \int_{0}^{t_{i}}\left\|f\left(\varrho, x_{n}(\varrho)\right)-f(\varrho, x(\varrho))\right\| d \varrho+\mathcal{M} \int_{0}^{t}\left\|f\left(\varrho, x_{n}(\varrho)\right)-f(\varrho, x(\varrho))\right\| d \varrho \\
\rightarrow 0 \text { as } n \rightarrow \infty .
\end{gathered}
$$

Thus $Q$ is continuous map on $D$.

Next, we will prove $\mathcal{Q}: D \rightarrow D$ is monotone increasing. Let $x_{1}, x_{2} \in D, x_{1} \leqslant x_{2}$. Since $\mathbb{U}(t, s)$ is positive evolution system and the hypothesis $(H 2)$ holds, it is easy to see $2 x_{1} \leqslant 2 x_{2}$. Suppose $\omega_{0}^{\prime}(\eta)+\mathbb{A}(\eta) \omega_{0}(\eta)=$ $h(\eta)$, Definition 2.4, implies $h(\eta) \leqslant f\left(\eta, \omega_{0}(\eta)\right)$, for $\eta \in J$. From Lemma 3.2 and the positivity of evolution system, we have

$$
\begin{aligned}
\omega_{0}(\eta) & =\mathbb{U}(\eta, 0) \mathcal{B} x_{0}+\sum_{i=1}^{k} \xi_{i} \mathbb{U}(\eta, 0) \mathcal{B} \int_{0}^{t_{i}} \mathbb{U}\left(t_{i}, s\right) h(s) d s+\int_{0}^{\eta} \mathbb{U}(\eta, s) h(s) d s \\
& \leqslant \mathbb{U}(\eta, 0) \mathcal{B} x_{0}+\sum_{i=1}^{k} \xi_{i} \mathbb{U}(\eta, 0) \mathcal{B} \int_{0}^{t_{i}} \mathbb{U}\left(t_{i}, s\right) f\left(s, \omega_{0}(s)\right) d s+\int_{0}^{\eta} \mathbb{U}(\eta, s) f\left(s, \omega_{0}(s)\right) d s \\
& =Q \omega_{0}(\eta) .
\end{aligned}
$$

Hence, $\omega_{0} \leqslant 2 \omega_{0}$. In the same way, we get $2 v_{0} \leqslant v_{0}$. For $u \in D$, we have $\omega_{0} \leqslant 2 \omega_{0} \leqslant 2 u \leqslant 2 v_{0} \leqslant v_{0}$. Therefore, $Q: D \rightarrow D$ is monotone increasing. 
Now we will show $Q(D)$ is equicontinuous on $J$. For $x \in D$ and $\eta_{1}, \eta_{2} \in J$ with $\eta_{1}<\eta_{2}$ we have

$$
\begin{aligned}
\left\|Q \mathcal{Q} x\left(\eta_{2}\right)-\mathcal{Q} x\left(\eta_{1}\right)\right\| \leqslant & \left\|\mathbb{U}\left(\eta_{2}, 0\right)-\mathbb{U}\left(\eta_{1}, 0\right)\right\|\|\mathcal{B}\|\left\|x_{0}\right\| \\
& +\|\mathcal{B}\|\left(\sum_{i=1}^{k} \xi_{i} \int_{0}^{t_{i}}\left\|\mathbb{U}\left(t_{i}, \varrho\right)\right\|\|f(\varrho, x(\varrho))\| d \varrho\right)\left\|\mathbb{U}\left(\eta_{2}, 0\right)-\mathbb{U}\left(\eta_{1}, 0\right)\right\| \\
& +\int_{0}^{\eta_{1}}\left\|\mathbb{U}\left(\eta_{2}, \varrho\right)-\mathbb{U}\left(\eta_{1}, \varrho\right)\right\|\|f(\varrho, x(\varrho))\| d \varrho+\int_{\eta_{1}}^{\eta_{2}}\left\|\mathbb{U}\left(\eta_{2}, \varrho\right)\right\|\|f(\varrho, x(\varrho))\| d \varrho \\
\leqslant & \left\|\mathbb{U}\left(\eta_{2}, 0\right)-\mathbb{U}\left(\eta_{1}, 0\right)\right\|\|\mathcal{B}\|\left\|x_{0}\right\| \\
& +\mathcal{M}\|\mathcal{B}\| b c\left(\sum_{i=1}^{k} \xi_{i}\right)\left\|\mathbb{U}\left(\eta_{2}, 0\right)-\mathbb{U}\left(\eta_{1}, 0\right)\right\| \\
& +\int_{0}^{\eta_{1}}\left\|\mathbb{U}\left(\eta_{2}, \varrho\right)-\mathbb{U}\left(\eta_{1}, \varrho\right)\right\|\|f(\varrho, x(\varrho))\| d \varrho+\mathcal{M} c\left(\eta_{2}-\eta_{1}\right) . \\
& I_{1}+I_{2}+I_{3}+I_{4} .
\end{aligned}
$$

For $\eta_{1}=0$, it is easy to see that $I_{3}=0$. For $\eta_{1}>0$ and $\epsilon>0$ small enough, we obtain

$$
\begin{aligned}
I_{3} & \leqslant \int_{0}^{\eta_{1}-\epsilon}\left\|\mathbb{U}\left(\eta_{2}, \varrho\right)-\mathbb{U}\left(\eta_{1}, \varrho\right)\right\|\|f(\varrho, x(\varrho))\| d \varrho+\int_{\eta_{1}-\epsilon}^{\eta_{1}}\left\|\mathbb{U}\left(\eta_{2}, \varrho\right)-\mathbb{U}\left(\eta_{1}, \varrho\right)\right\|\|f(\varrho, x(\varrho))\| d \varrho \\
& \leqslant c\left(\eta_{1}-\epsilon\right) \sup _{\varrho \in\left[0, \eta_{1}-\epsilon\right]}\left\|\mathbb{U}\left(\eta_{2}, \varrho\right)-\mathbb{U}\left(\eta_{1}, \varrho\right)\right\|+2 \mathcal{M} c \epsilon \\
& \rightarrow 0 \text { as } \eta_{2} \rightarrow \eta_{1}, \epsilon \rightarrow 0,
\end{aligned}
$$

using the continuity of $\{U(\eta, \varrho): \eta>\varrho\}$ in uniform operator topology. By using Lemma 2.2, it is easy to see that $I_{1} \rightarrow 0, I_{2} \rightarrow 0$ as $\eta_{2} \rightarrow \eta_{1}$. Also it is clear from the expression of $I_{4}$ that $I_{4} \rightarrow 0$ as $\eta_{2} \rightarrow \eta_{1}$. As a result $\left\|Q x\left(\eta_{2}\right)-Q x\left(\eta_{1}\right)\right\| \rightarrow 0$ as $\eta_{2} \rightarrow \eta_{1}$, independently of $x \in D$. Hence $Q(D)$ is equicontinuous on $J$.

Now we define the sequences

$$
\omega_{n}=Q \omega_{n-1} \quad \text { and } \quad v_{n}=2 v_{n-1}, \quad n \in \mathbb{N},
$$

monotonicity of $Q$ implies

$$
\omega_{0} \leqslant \omega_{1} \leqslant \cdots \omega_{n} \leqslant \cdots \leqslant v_{n} \leqslant \cdots \leqslant v_{1} \leqslant v_{0}
$$

Let $S=\left\{\omega_{n}\right\}$ and $S_{0}=\left\{\omega_{n-1}\right\}$. Then $S_{0}=S \cup\left\{\omega_{0}\right\}$ and $\mu\left(S_{0}(t)\right)=\mu(S(t)), t \in J$. Since the sequence $\left\{\omega_{n}(0)\right\}$ is convergent, so $\mu\left(\left\{\omega_{n}(0)\right\}\right)=0$. From Lemma 2.9, (H3), (3.9) and (3.11), we get

$$
\begin{aligned}
\mu(S(t)) & =\mu\left(\mathcal{Q}\left(S_{0}(t)\right)\right) \\
& =\mu\left(\left\{\mathbb{U}(t, 0) \mathcal{B} x_{0} \sum_{i=1}^{k} \xi_{i} \mathbb{U}(t, 0) \mathcal{B} \int_{0}^{t_{i}} \mathbb{U}\left(t_{i}, s\right) f\left(s, \omega_{n-1}(s)\right) d s+\int_{0}^{t} \mathbb{U}(t, s) f\left(s, \omega_{n-1}(s)\right) d s\right\}\right) \\
& \leqslant \mathcal{M} \mu\left(\left\{\omega_{n}(0)\right\}\right)+2 \mathcal{M} \int_{0}^{t} \mu\left(f\left(s, \omega_{n-1}(s)\right)\right) d s \\
& \leqslant 2 \mathcal{M L} \mathcal{L} \int_{0}^{t} \mu\left(\left\{\omega_{n-1}(s)\right\}\right) d s \\
& \leqslant 2 \mathcal{N} \mathcal{L} \int_{0}^{t} \mu\left(S_{0}(s)\right) d s \\
& 2 \mathcal{M L} \int_{0}^{t} \mu(S(s)) d s .
\end{aligned}
$$


Since $\left\{Q \omega_{n-1}\right\}$, i.e. $\left\{\omega_{n}\right\}$ is equicontinuous, by Lemma 2.8 and (3.13), we obtain

$$
\begin{aligned}
\mu(S) & =\sup _{t \in J} \mu(S(t)) \\
& \leqslant 2 \mathcal{N} \mathcal{L} b \sup _{t \in J} \mu(S(t))=2 \mathcal{M} \mathcal{L} b \mu(S)=K \mu(S) .
\end{aligned}
$$

Since $K<1$, therefore $\mu(S)=0$. Hence the set $S$ is relatively compact in $D$, so there is a convergent subsequence of $\left\{\omega_{n}\right\}$ in $D$. From (3.12), it is easy to see that $\left\{\omega_{n}\right\}$ itself is a convergent sequence. Let $\omega_{n} \rightarrow \omega^{\star}$ as $n \rightarrow \infty$. By (3.9) and (3.11)

$$
\begin{aligned}
\omega_{n}(t) & =2 \omega_{n-1}(t) \\
& =\mathbb{U}(t, 0) \mathcal{B} x_{0}+\sum_{i=1}^{k} \xi_{i} \mathbb{U}(t, 0) \mathcal{B} \int_{0}^{t_{i}} \mathbb{U}\left(t_{i}, s\right) f\left(s, \omega_{n-1}(s)\right) d s+\int_{0}^{t} \mathbb{U}(t, s) f\left(s, \omega_{n-1}(s)\right) d s .
\end{aligned}
$$

Let $n \rightarrow \infty$ and using Lebesgue dominated convergence theorem, we get $\omega^{\star}=Q \omega^{\star}$ and $\omega^{\star} \in \mathbf{C}(J, X)$. Hence $\omega^{\star}$ is a mild solution for (1.1)-(1.2). In the same way there is $v^{\star} \in \mathbf{C}(J, X)$ with $v_{n} \rightarrow v^{\star}$ as $n \rightarrow \infty$, and $v^{\star}=Q v^{*}$. If $x \in D$ and $x=Q 2 x$, then $\omega_{1}=2 \omega_{0} \leqslant Q x=x \leqslant Q v_{0}=v_{1}$. From the process of induction $\omega_{n} \leqslant x \leqslant v_{n}$, and $\omega_{0} \leqslant \omega^{*} \leqslant x \leqslant v^{*} \leqslant v_{0}$ as $n \rightarrow \infty$. That means $\omega^{*}$ is the minimal and $v^{*}$ is the maximal mild solution for (1.1)-(1.2) in $\left[\omega_{0}, v_{0}\right]$.

Corollary 3.4. Suppose $X$ is a partially ordered and weakly sequentially complete Banach space with normal positive cone $\mathcal{P}$, the assumptions (H0), (H1), (H2) and (A1)-(A3) hold, $f$ is continuous from $J \times X \rightarrow X, x_{0} \in X$, and $\omega_{0}, v_{0} \in C^{1}(J, X)$ are lower and upper solutions respectively for (1.1)-(1.2), then the system (1.1)-(1.2) has extremal mild solutions in the interval $\left[\omega_{0}, v_{0}\right]$.

Proof. Since the assumptions (H1), (H2) hold, by the proof of Theorem 3.3 we have the sequences $\left\{\omega_{n}(t)\right\}$ and $\left\{v_{n}(t)\right\}$ defined by (3.11) satisfying (3.12). So, $\left\{\omega_{n}(t)\right\}$ and $\left\{v_{n}(t)\right\}$ are monotone and bounded, hence are precompact on $X$ for $X$ is weakly sequentially complete Banach space. Therefore, the sequences are convergent uniformly. Let $\omega^{\star}(t)=\lim _{n \rightarrow \infty} \omega_{n}(t)$ and $v^{\star}(t)=\lim _{n \rightarrow \infty} v_{n}(t)$, for $t \in J$. By Lebesgue dominated convergence theorem, we get $\omega^{\star}=2 \omega^{\star}$ and $v^{\star}=2 v^{\star}$ with $\omega^{\star}, v^{\star} \in \mathbf{C}(J, X)$. Hence $\omega^{\star}$ and $v^{\star}$ are a mild solutions for (1.1)(1.2). If $x \in D$ and $x=2 x$, then $\omega_{1}=2 \omega_{0} \leqslant Q x=x \leqslant Q v_{0}=v_{1}$. From the process of induction $\omega_{n} \leqslant x \leqslant v_{n}$, and $\omega_{0} \leqslant \omega^{\star} \leqslant x \leqslant v^{\star} \leqslant v_{0}$ as $n \rightarrow \infty$. That means $\omega^{\star}$ is the minimal and $v^{\star}$ is the maximal mild solution for (1.1)-(1.2).

Theorem 3.5. Suppose $X$ is a partially ordered complete norm space, with normal positive cone $\mathcal{P}$ and normal constant $\mathcal{N}$, the assumptions (H0), (H1), (H2), (H4) and (A1)-(A3) hold, $f \in \mathbf{C}(J \times X, X), x_{0} \in X$, and $\omega_{0}, v_{0} \in$ $C^{1}(J, X)$ are lower and upper solutions respectively for (1.1)-(1.2). Moreover, assume the following:

(H5) There is a constant $\mathcal{L}_{1}>0$ such that, for $t \in J$, and $z_{1}, z_{2} \in X$ with $\omega_{0}(t) \leqslant z_{1} \leqslant z_{2} \leqslant v_{0}(t)$,

$$
f\left(t, z_{2}\right)-f\left(t, z_{1}\right) \leqslant \mathcal{L}_{1}\left(z_{2}-z_{1}\right) .
$$

Then, the system (1.1)-(1.2) has a unique mild solution in $\left[\omega_{0}, v_{0}\right]$, provided that

$$
K_{1}:=\frac{2-\mathcal{M} \sum_{i=1}^{k} \xi_{i}}{1-\mathcal{M} \sum_{i=1}^{k} \xi_{i}} \mathcal{N} \mathcal{L}_{1} \mathcal{M} b b<1 .
$$

Proof. Let $\left\{x_{n}\right\} \subset X$ be an increasing monotone sequence, and $n, m \in \mathbb{N}$ with $n>m$. (H2) and (H5) imply

$$
0 \leqslant f\left(t, x_{n}\right)-f\left(t, x_{m}\right) \leqslant \mathcal{L}_{1}\left(x_{n}-x_{m}\right) .
$$


From the normality of $\mathcal{P}$

$$
\left\|f\left(t, x_{n}\right)-f\left(t, x_{m}\right)\right\| \leqslant \mathcal{N} \mathcal{L}_{1}\left\|\left(x_{n}-x_{m}\right)\right\| .
$$

So by Lemma 2.7, we get

$$
\mu\left(\left\{f\left(t, x_{n}\right)\right\}\right) \leqslant \mathcal{N} \mathcal{L}_{1} \mu\left(\left\{x_{n}\right\}\right) .
$$

Hence the assumption (H3) holds, and Theorem 3.3 is applicable. Therefore, (1.1)-(1.2) has minimal mild solution $\omega^{\star}$ and maximal mild solutions $v^{\star}$ in $\left[\omega_{0}, v_{0}\right]$. By (3.9), (H5), and the positivity of the operator $\mathbb{U}(t, s)$, we get

$$
\begin{aligned}
0 & \leqslant v^{\star}(t)-\omega^{\star}(t)=Q v^{\star}(t)-Q \omega^{\star}(t) \\
& =\sum_{i=1}^{k} \xi_{i} \mathbb{U}(t, 0) \mathcal{B} \int_{0}^{t_{i}} \mathbb{U}\left(t_{i}, s\right)\left[f\left(s, v^{\star}(s)\right)-f\left(s, \omega^{\star}(s)\right)\right] d s+\int_{0}^{t} \mathbb{U}(t, s)\left[f\left(s, v^{\star}(s)\right)-f\left(s, \omega^{\star}(s)\right)\right] d s \\
& \leqslant \mathcal{L}_{1}\left[\sum_{i=1}^{k} \xi_{i} \mathbb{U}(t, 0) \mathcal{B} \int_{0}^{t_{i}} \mathbb{U}\left(t_{i}, s\right)\left(v^{\star}(s)-\omega^{\star}(s)\right) d s+\int_{0}^{t} \mathbb{U}(t, s)\left(v^{\star}(s)-\omega^{\star}(s)\right) d s\right] .
\end{aligned}
$$

Now using (H1), (3.3), and the normality of positive cone, we get

$$
\begin{aligned}
\left\|v^{*}-\omega^{*}\right\| \leqslant & \mathcal{N} \mathcal{L}_{1}\left[\left(\sum_{i=1}^{k} \xi_{i}\right) \mathcal{M} \mathcal{N}^{2}\|\mathcal{B}\| \int_{0}^{b}\left\|v^{*}(s)-\omega^{\star}(s)\right\| d s+\mathcal{M} \int_{0}^{b}\left\|v^{\star}(s)-\omega^{*}(s)\right\| d s\right] \\
\leqslant & \mathcal{N} \mathcal{L}_{1} \mathcal{M} b(\|\mathcal{B}\|+1)\left\|v^{\star}-\omega^{\star}\right\| \\
\leqslant & \frac{2-\mathcal{M} \sum_{i=1}^{k} \xi_{i}}{1-\mathcal{M} \sum_{i=1}^{k} \xi_{i}} \mathcal{N} \mathcal{L}_{1} \mathcal{M} b\left\|v^{\star}-\omega^{\star}\right\| \\
= & K_{1}\left\|v^{\star}-\omega^{\star}\right\| .
\end{aligned}
$$

Since $K_{1}<1$, so $\left\|v^{\star}-\omega^{\star}\right\|=0$, i.e. $v^{\star}(t)=\omega^{\star}(t)$ for all $t \in J$. Hence $v^{\star}=\omega^{\star}$ is the unique mild solution for (1.1)-(1.2) in $\left[\omega_{0}, v_{0}\right]$.

\section{Example}

Now we consider an example to show how our abstract results can be applied to a concrete problem. Consider the following partial differential equation:

$$
\left\{\begin{array}{l}
x^{\prime}(t, z)+a(t, z) \frac{\partial^{2}}{\partial z^{2}} x(t, z)=\frac{1}{4} t^{2} x(t, z), \quad z \in[0, \pi], \quad t \in J=[0, b] \\
x(t, 0)=0, \quad x(t, \pi)=0, \quad t \in J ; \\
x(0, z)=\sum_{i=1}^{k} \xi_{i} x\left(t_{i}, z\right)+x_{0}(z), \quad z \in[0, \pi],
\end{array}\right.
$$

where $X=L^{2}([0, b] \times[0, \pi], \mathbb{R}), x_{0}(z) \in X, a(t, z)$ is continuous function and satisfies uniformly Hölder continuity in $t$, and $0<t_{1}<t_{2}<\ldots<t_{i}<b, \xi_{i} \neq 0(i=1,2, \ldots k)$ are real numbers. Define

$$
\mathbb{A}(t) x(t, z)=a(t, z) \frac{\partial^{2}}{\partial z^{2}} x(t, z),
$$

with domain $D(\mathbb{A})=\left\{x \in X: x, \frac{\partial x}{\partial z}\right.$ are absolutely continuous, $\left.\frac{\partial^{2} x}{\partial z^{2}} \in X, x(0)=x(\pi)=0\right\}$. It is well known that $-\mathbb{A}(t)$ generates a positive evolution system of bounded linear operators $\mathbb{U}(t, s)$ on $X$ with $\|\mathbb{U}(t, s) \leqslant \mathcal{M}\|$ and satisfy the conditions (A1)-(A3) (see [23]). Put $x(t)=x(t, \cdot)$ that is $x(t)(z)=x(t, z), t \in[0, b], z \in[0, \pi]$ and $f(t, x(t))(z)=\frac{1}{4} t^{2} x(t, z)$, then the system (4.1) can be rewritten into the abstract form of (1.1)-(1.2). It is easy to check that assumption $(H 2)$ holds. Now, assume the following: 
1. $x_{0}(z) \geqslant 0$ for $z \in[0, \pi]$, and there exists a function $v(t, z) \geqslant 0$ such that

$$
\begin{aligned}
v^{\prime}(t, z)+\mathbb{A}(t) v(t, z) & \geqslant \frac{1}{4} t^{2} v(t, z), \quad t \in J, z \in[0, \pi], \\
v(t, 0) & =v(t, \pi)=0, \quad t \in J, \\
v(0, z) & \geqslant \sum_{i=1}^{k} \xi_{i} v\left(t_{i}, z\right)+x_{0}(z), \quad z \in[0, \pi] .
\end{aligned}
$$

2. $\xi_{i}>0, i=1,2, \ldots, k$ and $\mathcal{M} \sum_{i=1}^{k} \xi_{i}<1$.

From the above assumptions it is clear that $(H 1)$ holds, and $\omega_{0}=0$ and $v_{0}=v(t, z)$ are the lower and upper solutions for the system (4.1). So, by Corollary 3.4, we conclude that the minimal and maximal mild solutions for (4.1) exist between the lower solution 0 and upper solution $v$.

Acknowledgments: The authors would like to express thanks to the editor and referees for their careful reading of the manuscript and valuable comments. The work of first author is supported by Ministry of Human Resource Development, India with grant code “ MHR-01-23-200-428”.

\section{References}

[1] Byszewski L., Theorem about the existence and uniqueness of solutions of a semilinear evolution nonlocal Cauchy problem, J. Math. Anal. Appl., 1991, 162(2), 494-505

[2] Deng K., Exponential decay of solutions of semilinear parabolic equations with nonlocal initial conditions, J. Math. Anal. Appl., 1993, 179(2), 630-637

[3] Chen P., Zhang X., Li Y., Approximation technique for fractional evolution equations with nonlocal integral conditions, Mediterr. J. Math., 2017, 14(6), 226

[4] Du S., Lakshmikantam V., Monotone iterative technique for differential equation in a Banach space, J. Math. Anal. Appl., 1982, 87(2), 454-459

[5] Chen P., Li Y., Monotone iterative technique for a class of semilinear evolution equations with nonlocal conditions, Results Math., 2013, 63(3-4), 731-744

[6] Chen P., Mu J., Monotone iterative method for semilinear impulsive evolution equations of mixed type in Banach space, Electron. J. Differ. Equ., 2010, 149, 1-13

[7] Mu J., Monotone iterative technique for fractional evolution equations in Banach spaces, J. Appl. Math., 2011, Article ID 767186, DOI: 10.1155/2011/767186

[8] Mu J., Li Y., Monotone iterative technique for impulsive fractional evolution equations, J. Inequal. Appl., 2011, 125, 1-12

[9] Chen P., Li Y., Yang H., Perturbation method for nonlocal impulsive evolution equations, Nonlinear Anal. Hybrid Syst., 2013, $8,22-30$

[10] Chen P., Li Y., Existence of mild solutions for fractional evolution equations with mixed monotone nonlocal condition, $\mathrm{Z}$. Angew. Math. Phys., 2014, 65(4), 711-728

[11] Jeet K., Bahuguna D., Monotone iterative technique for nonlocal fractional differential equations with finite delay in a Banach space, Electron. J. Qual. Theory Differ. Equ., 2015, 9, 1-16

[12] Chaudhary R., Pandey D. N., Monotone iterative technique for neutral fractional differential equation with infinite delay, Math. Methods Appl. Sci., 2016, 39(15), 4642-4653

[13] Chen P., Zhang X., Li Y., Iterative method for a new class of evolution equations with non-instantaneous impulses, Taiwanese J. Math., 2017, 21(4), 913-942

[14] Yan Z., On solutions of semilinear evolution integro-differential equations with nonlocal conditions, Tamkang J. Math., 2009, 40(3), 257-269

[15] Haloi R., Pandey D. N., Bahuguna, D., Bahuguna, D., Existence uniqueness and asymptotic stability of solutions to nonautonomous semi-linear differential equations with deviated arguments, Nonlinear Dyn. Syst. Theory, 2012, 12(2), 179-191

[16] Chadha A., Pandey D. N., Mild solutions for non-autonomous impulsive semi-linear differential equations with iterated deviating arguments, Electron. J. Differ. Equ., 22015, 2015(222), 1-14

[17] El-Borai M. M., The fundamental solutions for fractional evolution equations of parabolic type, Bol. Asoc. Mat. Venez., 2004, $\mathrm{XI}(1), 29-43$

[18] Li F., Existence for non-autonomous fractional integro-differential equations with nonlocal conditions, Abstr. Appl. Anal., 2013, Article ID 723453, DOI: 10.1155/2013/723453 
[19] Chen P., Zhang X., Li Y., Study on fractional non-autonomous evolution equations with delay, Comput. Math. Appl., 2017, 73(5), 794-803

[20] Benchohra M., Neito J. J., Rezoug N., Second order evolution equations with nonlocal conditions, Demonstr. Math., 2017, 50, 309-319

[21] Chen P., Zhang X., Li Y., Fractional non-autonomous evolution equation with nonlocal conditions, J. Pseudo-Differ. Oper. Appl., DOI: 10.1007/s11868-018-0257-9

[22] Chen P., Zhang X., Li Y., A blowup alternative result for fractional non-autonomous evolution equation of Volterra type, Commun. Pure Appl. Anal., 2018, 17(5), 1975-1992

[23] Pazy A., Semigroup of Linear Operators and Applications to Partial Differential Equations, Applied Mathematical Sciences, Vol. 44, Springer-Verlag, New York, 1983

[24] Friedman A., Partial Differential Equations, Dover Publication, New York, 1997

[25] Heinz H. P., On the behaviour of noncompactness with respect to differentiation and integration of vector valued functions, Nonlinear Anal., 1983, 7(12), 1351-1371

[26] Deimling K., Nonlinear Functional Analysis, Springer-Verlag, Berlin, 1985 